\title{
DIGITAL BREAST TOMOSYNTHESIS AND FULL-FIELD DIGITAL MAMMOGRAPHY IN BREAST CANCER DETECTION ASSOCIATED WITH FOUR ASYMMETRY TYPES
}

DOI: $10.36740 /$ WLek202104106

\author{
Andrii V. Gurando ${ }^{1,4}$, Tetiana M. Babkina ${ }^{2}$, Iryna M. Dykan', , Tetiana M. Kozarenko², Viacheslav R. Gurando ${ }^{3}$, Vadim V. Telniy $^{4}$ \\ 'SI, "INSTITUTE OF NUCLEAR MEDICINE AND DIAGNOSTIC RADIOLOGY OF NATIONAL ACADEMY OF MEDICAL SCIENCE OF UKRAINE”, KYIV, UKRAINE \\ 2P.L. SHUPYK NATIONAL MEDICAL ACADEMY OF POSTGRADUATE EDUCATION, KYIV, UKRAINE \\ 3UZHHOROD NATIONAL UNIVERSITY, UZHHOROD, UKRAINE \\ ${ }^{4}$ MEDICAL CENTER, «VERUM EXPERT», KYIV, UKRAINE
}

\begin{abstract}
The aim: Comparing sensitivity and specificity of digital breast tomosynthesis and full-field digital mammography in breast cancer detection associated with four different types of asymmetries according to BI-RADS Atlas.

Materials and methods: Study included 201 patients with four types of asymmetries according BI-RADS atlas (asymmetry - 81 (40,3\%), focal asymmetry - 82 (40,8\%), global asymmetry - $36(17,9 \%)$ and developing asymmetry - $2(1,0 \%))$ who underwent full-field digital mammography, digital breast tomosynthesis and hand-held full breast ultrasound from January 2017 to June 2018.

The general rate of breast cancer for the 201 patients with asymmetries was 8 cases $(4,0 \%)(I B C, n=6(3,0 \%) ; D C I S, n=2(1,0 \%)$ other findings associated with asymmetries were non-malignant, $n=10(5,0 \%)$ (sclerosing adenosis, $n=5(2,5 \%)$; fibroadenomatosis, $n=3(1,5 \%)$; simple cyst, $n=1(0,5 \%)$; radial scar associated with papilloma, typical ductal hyperplasia and sclerosing adenosis, $n=1(0,5 \%)$.

Results: Analysis of the results showed that sensitivity of digital breast tomosynthesis was $75.0 \%$ [95\% Cl, 34.91\% to 96.81\%] and specificity was $94.8 \%[95 \%$ Cl, $90.68 \%$ to 97.49\%] which was superior to full-field digital mammography sensitivity $50.0 \%$ [95\% Cl, $15.70 \%$ to $84.30 \%]$ and specificity $91.19 \%$ [95\% Cl, $86.27 \%$ to $94.78 \%]$ for breast cancer detection associated with different types of asymmetries.

Conclusions: Using of digital breast tomosynthesis in assessment of breast asymmetries can improve sensitivity and specificity in breast cancer detection and reduce number of unnecessary biopsies and short-interval follow-up examinations.
\end{abstract}

KEY WORDS: Asymmetries, digital breast tomosynthesis, mammography

Wiad Lek. 2021;74(4):842-848

\section{INTRODUCTION}

According to the current data of national cancer-register breast cancer (BC) takes the first place among oncologic diseases in Ukrainian woman [1].

Large studies showed that screening mammography can reduce $\mathrm{BC}$ mortality in population approximately for $40 \%$ [2-4]. But dense fibroglandular breast tissue, due to its overlapping effect, still reduces sensitivity of full-field digital mammography (FFDM) to $48.0 \%-62.9 \%$ in dense breasts in comparison with high FFDM sensitivity for fatty breasts $85.7 \%-88.8 \%$ [5-7].

Corresponding to the last investigations - digital breast tomosynthesis (DBT) can improve BC detection in women with dense breasts and reduce recall rate, especially for asymmetries, due to $3 \mathrm{D}$ visualization of breast patterns [8-12].

Although there is clearly wide variation in breast size and parenchymal pattern, the breasts are generally symmetric structures with similar density and architecture
[13]. However, asymmetries account for $6 \%$ of non-palpable, screen-detected cancers, represent $27 \%$ of missed cancers and are the second most common cause of false negative screening recall assessments [14-17].

The term asymmetric breast tissue refers to a greater volume or density of breast tissue in one breast than in the corresponding area in the contralateral breast, in contrast to the mass, which is three-dimensional, asymmetric findings is often normal fibroglandular tissue with interspersed fat and lacking convex margins, which is caused by the superimposition artifact in a given mammographic projection, but, it can be possible that the asymmetry, in fact, is a cancer $[13,14,18]$.

The last 5-th edition of Breast Imaging - Report and Data System Atlas (BI-RADS) divided asymmetric breast densities into four categories: asymmetry, focal asymmetry, global asymmetry and developing asymmetry [18].

Detection and diagnostic evaluation of asymmetries is particularly challenging, as many appear similar to 
Table I. Patients with four types of asymmetries assessed, by using BI-RADS categories, separately for FFDM and for DBT.

\begin{tabular}{|c|c|c|c|c|c|c|c|c|c|c|}
\hline \multirow{2}{*}{ Asymmetry type } & \multicolumn{5}{|c|}{ BI-RADS categories for FFDM } & \multicolumn{5}{|c|}{ BI-RADS categories for DBT } \\
\hline & 1 & 2 & 3 & 4 & 5 & 1 & 2 & 3 & 4 & 5 \\
\hline One-view asymmetry & 0 & 23 & 50 & 8 & 0 & 12 & 55 & 10 & 4 & 0 \\
\hline Focal asymmetry & 0 & 17 & 53 & 12 & 0 & 0 & 52 & 19 & 7 & 3 \\
\hline Global asymmetry & 0 & 36 & 0 & 0 & 0 & 0 & 36 & 0 & 0 & 0 \\
\hline Developing asymmetry & 0 & 0 & 0 & 2 & 0 & 0 & 0 & 0 & 2 & 0 \\
\hline
\end{tabular}

Table II. ACR breast density distribution for each asymmetry type.

\begin{tabular}{|c|c|c|c|c|c|}
\hline \multirow{2}{*}{ Asymmetry type } & \multicolumn{4}{|c|}{ ACR density types } & \multirow[b]{2}{*}{ total } \\
\hline & $\mathbf{a}$ & b & c & d & \\
\hline One-view asymmetry & 0 & 28 & 51 & 2 & 81 \\
\hline Focal asymmetry & 0 & 37 & 45 & 0 & 82 \\
\hline Global asymmetry & 0 & 5 & 30 & 1 & 36 \\
\hline Developing asymmetry & 0 & 0 & 2 & 0 & 2 \\
\hline total & 0 & 70 & 128 & 3 & 201 \\
\hline
\end{tabular}

Table III. Breast cancer involvement depending on the type of asymmetry.

\begin{tabular}{cccc}
\hline Asymmetry type & Number of cases & Biopsy performed & Ca detected \\
\hline One-view asymmetry & 81 & 4 & 1 \\
\hline Focal asymmetry & 82 & 12 & 6 \\
\hline Global asymmetry & 36 & 0 & 0 \\
\hline Developing asymmetry & 2 & 2 & 1 \\
\hline
\end{tabular}

fibroglandular tissue at mammography [19]. At mammographic examinations they have low but clinically important yield of invasive cancer and are common source of false-positive results, particularly among women younger than 70 years, but that not all cancers present with the 'typical' features of malignancy $[14,20]$. Asymmetry which cannot be attributed to a benign cause should undergo biopsy to avoid a delayed diagnosis of breast cancer [14].

\section{THE AIM}

The aim of our study was to compare sensitivity and specificity of DBT and FFDM in BC detection associated with different types of asymmetries.

\section{MATERIALS AND METHODS}

Study included 201 patients with four types of asymmetries according to BI-RADS atlas (asymmetry - 81 (40,3\%), focal asymmetry - 82 (40,8\%), global asymmetry - $36(17,9 \%)$ and developing asymmetry - $2(1,0 \%))$ who underwent FFDM, DBT and hand-held full breast ultrasound (HHUS) from January 2017 to June 2018. All asymmetries were assessed by using BI-RADS categories separately for FFDM and for DBT [Table I].

Suspicious asymmetries underwent morphological verification, $n=18(9,0 \%)$, probably benign and benign asymmetries were followed up for 24-30 months after the first mammographic study to ensure long-term stability of what is almost certainly benign pathology, $n=183(91 \%)$. During this time none of the patients underwent biopsy on the basis of one-view asymmetry and global asymmetry, while 2 developing asymmetries, which were focal asymmetries at the first examination, underwent $\mathrm{CNB}$ and one of it revealed DCIS.

Exclusion criteria for our study were: absence of asymmetries, lack of morphological verification for suspicious findings or absence of 2 years follow up for benign and probably benign findings, previous history of surgery or trauma concordant with asymmetry.

Bilateral FFDM and DBT were performed in two standard projections (CC and MLO) on Selenia Dimensions Mammography system (Hologic, USA). Stereotactic biopsies were performed on Hologic MultiCare Platinum Prone Breast Biopsy Table, when there wasn't possibility to establish correlation with HHUS and perform ultrasound-guided core needle biopsy (CNB).

HHUS and ultrasound-guided biopsies were performed on a Toshiba Viamo and Toshiba Aplio XG ultrasound systems with a linear probe centered at 9,0 MHz.

Age of the patients ranged from 37 years to 70 years with mean age of 45,4 years. Patient with suspicious asymmetries underwent 16 ultrasound-guided and 2 stereotactic $\mathrm{CNB}$, and revealed 8 malignant and 10 benign lesions.

Retrospectively, all studies with previously FFDM detected asymmetries, in consensus, were read by two dedicated breast radiologist. First only FFDM images in two standard projections (CC, MLO) were read and assessed by using 
BI-RADS categories, without knowing results of DBT examination and pathology, and then DBT images were assessed by using BI-RADS categories.

\section{RESULTS}

The overall rate of $\mathrm{BC}$ involvement for the 201 patients with asymmetries was 8 cases $(4,0 \%)$ (invasive breast cancers (IBC), $n=6(3,0 \%)$; ductal cancer in situ (DCIS), $n=2$ $(1,0 \%)$ other findings associated with asymmetries were non-malignant, $\mathrm{n}=10(5,0 \%)$ (sclerosing adenosis, $\mathrm{n}=5$ $(2,5 \%)$; fibroadenomatosis, $\mathrm{n}=3$ ( $1,5 \%)$; simple cyst, $\mathrm{n}=1$ $(0,5 \%)$; radial scar associated with papilloma, typical ductal hyperplasia and sclerosing adenosis, $\mathrm{n}=1(0,5 \%)$ [Table III].

Nine percent $(18 / 201)$ of the lesions were biopsied. We performed 16 ultrasound-guided CNB, while the remainder, when we couldn't find correlation between mammographic and sonographic picture, underwent stereotactic CNB (2/18) .

All $(n=36)$ global asymmetries were assessed identically by both methods, FFDM and DBT, BI-RADS-2, after ultrasound and 2 years of follow-up asymmetric fibroglandular tissue didn't change.

Among one-view asymmetries $(\mathrm{n}=81)$ we performed 4 biopsies and detected one BC.

Case Report №1. One-view asymmetry representing malignancy. (Fig.1) CC and (Fig.2) MLO views: a potential lesion lacking the characteristics of a mass is seen only on the (Fig.1) LCC view (red square), a corresponding abnormality is not seen on the (Fig.2) LMLO view. (Fig.3) RCC tomo scan view and US scan showed an irregular hypoechoic mass with indistinct margins and vascularity (red square) assessed as BI-RADS category 4. Pathologic diagnosis revealed ductal cancer in situ.

Due to DBT we avoided 4 unnecessary biopsies of benign breast findings which were stable during next 2 years, by lowering BI-RADS- 4 category to BI-RADS-3 category. Also we avoided short-time follow-up examinations in 40 cases by lowering BI-RADS-3 category to BI-RADS- 2 or even BI-RADS-1 category.

Case Report №2. One-view asymmetry representing summation artifact. (Fig.4) CC and (Fig.5) MLO views: asymmetry is seen only on the RCC view (red square). (Fig.6) On the RCC tomo scan view, the asymmetry didn't disappear. (Fig.6) Echo-scan, obtained for further evaluation, demonstrates an island of normal breast tissue that corresponds to the one-view asymmetry seen at FFDM. Follow-up mammography after 24 months showed no interval change.

We found $6 \mathrm{BC}$ and made 12 biopsies among 82 focal asymmetries.

Case Report №3. Focal asymmetry representing malignancy. (Fig.7) CC and (Fig.8) MLO views show a focal asymmetry in the retroareolar region of the right breast (red square). (Fig.7-8) RCC and RMLO tomo scan views detected an irregular mass with indistinct margins that was superimposed by fibroglandular breast tissue in the retroareolar region of the right breast. Pathology confirmed invasive carcinoma of no special type.
Three cases of BC represented as focal asymmetry were underestimated on FFDM and assessed as BI-RADS-3 finding. Five cases were overestimated by using BI-RADS- 4 category and lowers to BI-RADS- 3 category by using DBT. BI-RADS-3 category was used only in 19 cases after DBT examination instead of 53 cases during FFDM examination.

Case Report №4. Focal asymmetry representing summation shadow. (Fig.9) CC and (Fig.10) MLO views show a focal asymmetry in the right upper outer quadrant (red square). On the (Fig.9-10) RCC and RMLO tomo scans asymmetry lost its density and ultrasound (not shown) did not demonstrate any abnormal findings in the corresponding region. Follow-up mammography after 26 month showed no interval change.

We had only two cases of developing asymmetries which were focal asymmetries on initial FFDM and DBT examinations. In both cases, after negative dynamic were seen, findings were assessed as BI-RADS-4 lesions. One of these finding was ductal cancer in situ and other lesion was radial scar associated with papilloma, typical ductal hyperplasia and sclerosing adenosis.

The difference in assessment for BI-RADS- 2 and 3 categories for all types of asymmetries by using FFDM and DBT was statistically significant ([95\% CI, 12.27-22.08]; $p=0.00001$ ).

Analysis of the results showed that sensitivity of DBT was $75.0 \%$ [ $95 \%$ CI, $34.91 \%$ to $96.81 \%$ ] and specificity was $94.8 \%$ [ $95 \%$ CI, $90.68 \%$ to $97.49 \%$ ] which was superior to FFDM sensitivity $50.0 \%$ [ $95 \% \mathrm{CI}, 15.70 \%$ to $84.30 \%$ ] and specificity $91.19 \%$ [ $95 \%$ CI, $86.27 \%$ to $94.78 \%$ ] for BC detection associated with different types of asymmetries.

\section{DISCUSSION}

DBT is a relatively new modality for Ukrainian breast imaging. First experience of 3D mammography in Ukraine showed superior sensitivity for BC detection by using DBT than FFDM [21].

Another study showed that adding of DBT at screening mammography has lowered the number of callbacks of findings due to summation artifact and elevated the diagnostic accuracy of radiologists for BC detection [19].

In line with other studies, after FFDM and DBT we evaluated all asymmetries with targeted diagnostic HHUS and, in some cases, we performed spot-compression views on region of interest $[19,22,26$, ]. When we saw at ultrasound typical fibroglandular breast tissue which correlates with asymmetric breast finding, then patient was returned to routine follow-up mammography examinations. But when suspicious lesion was found in the area of interest, than $\mathrm{CNB}$ with US-guidance was performed. However, in 2 cases mammographically suspicious asymmetries were verified with stereotactic guidance, when we couldn't surely find correlation between asymmetric mammographic finding and ultrasound images in the region of interest.

According to the literature, asymmetry (area of fibroglandular-density tissue that is visible on only one mam- 


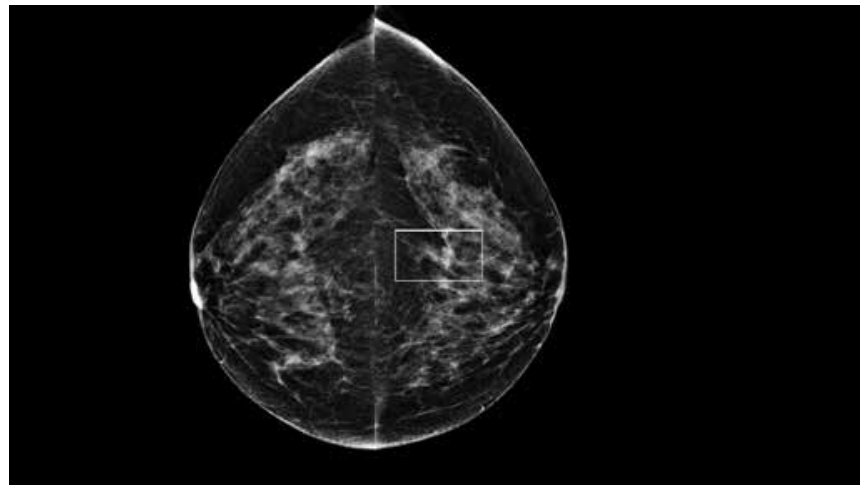

Fig. 1. RCC and LCC views

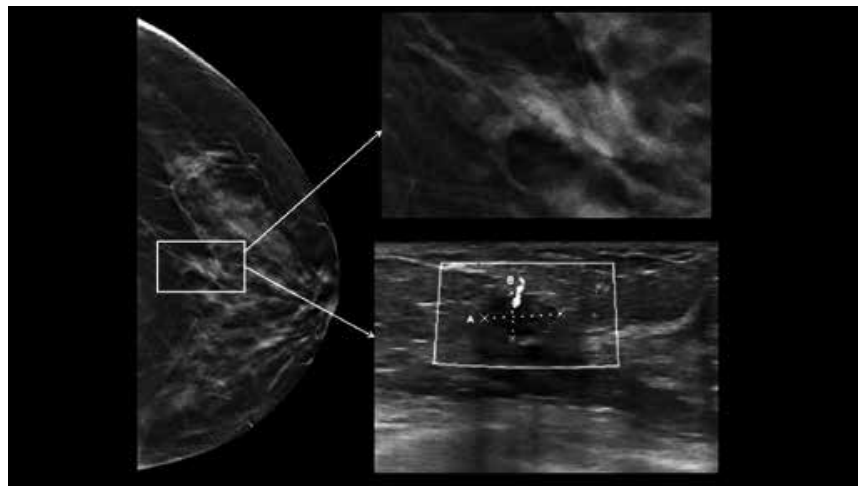

Fig. 3. Correlation between DBT images and US scan of suspicious lesion

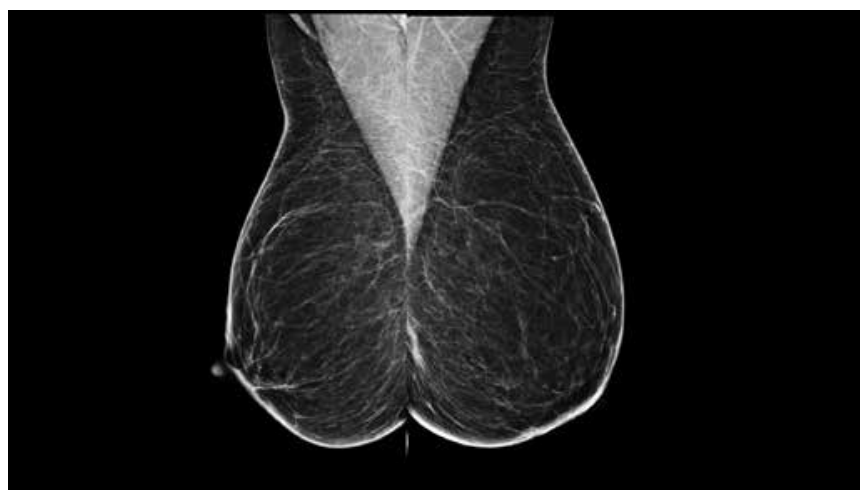

Fig. 5. RMLO and LMLO views

mographic projection) occurs 3,3\%-20,5\% of screening mammograms and its likelihood of malignancy is $1,8 \%$ $3,6 \%$, but radiologists often recall patients with abnormal one-view-only findings for additional imaging, because it can be cancer which is obscured by dense tissue on the other view or can be outside of image field [20]. Most of one-view-only findings represent fibroglandular tissue without any changes, and represents cancer very rare. Nevertheless, some asymmetries after additional examinations can be reclassified as mass or architectural distortion and increase breast cancer detection, in our study among 81 cases of one-view asymmetries we performed 4 biopsies and detected 1 invasive breast cancer [18-23].

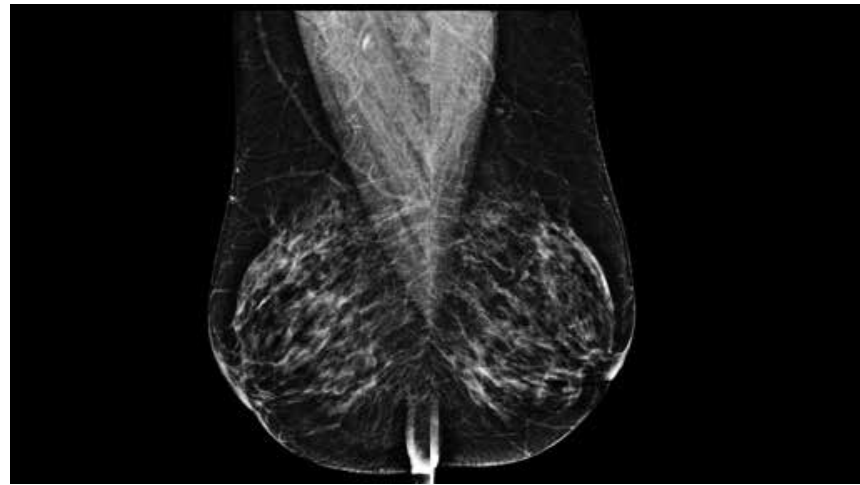

Fig. 2. RMLO and LMLO views

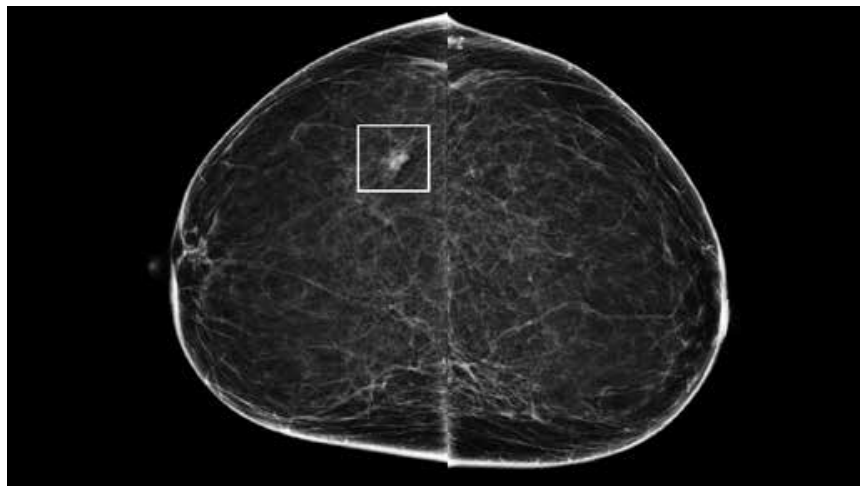

Fig. 4. $R C C$ and $L C C$ views - asymmetry is seen only on the right $C C$ view (red square)

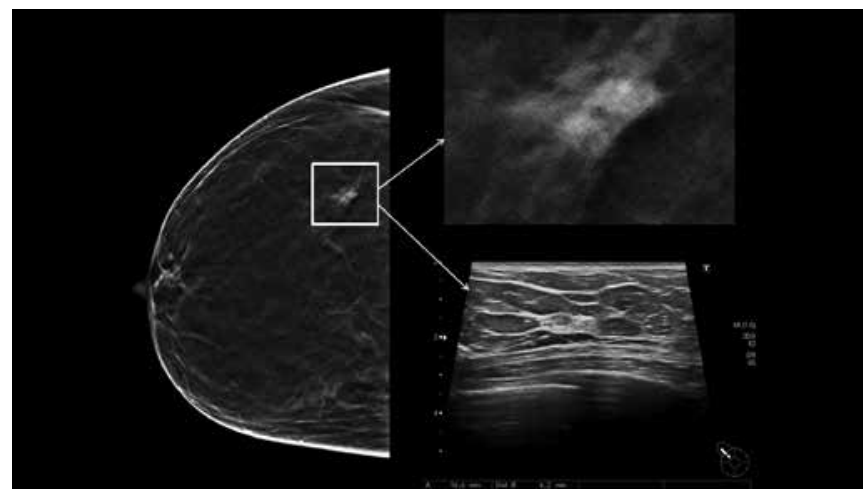

Fig. 6. Correlation between DBT images and US scan which demonstrates an island of normal breast tissue that corresponds to the asymmetry

Focal asymmetry - is judged relative to the corresponding location in the contralateral breast, and represents a relatively small amount of fibroglandular-density tissue over a confined portion of the breast (less than one quadrant) - occurs $0.87 \%-21.4 \%$ of screening mammograms, overall likelihood of malignancy of $0.67 \%-3.7 \%$, in our study among 82 cases of focal asymmetries we performed 12 biopsies and detected 6 cancers [18-20,24].

Global asymmetry - is judged relative to the corresponding area in the contralateral breast and represents a large amount of fibroglandular-density tissue over a substantial portion of the breast (at least one quadrant) and occurs about $3.0 \%$ of mammograms and should be considered 


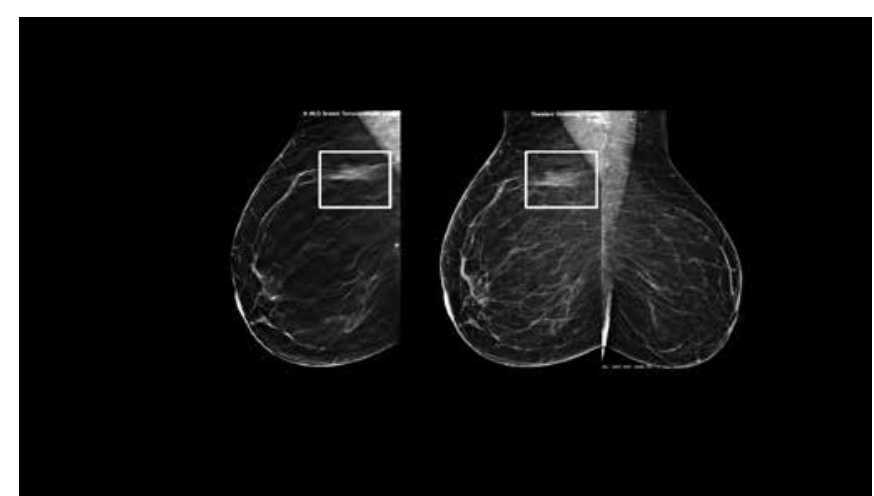

Fig. 7. RCC FFDM, DBT and LCC views - asymmetry is seen on the RCC views (red square)

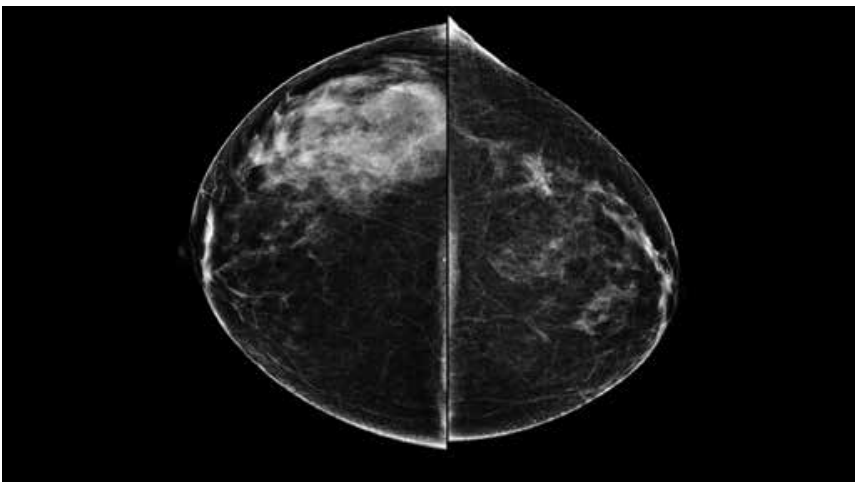

Fig. 9. RCC FFDM, DBT and LCC views - asymmetry is seen on the RCC views (red square)

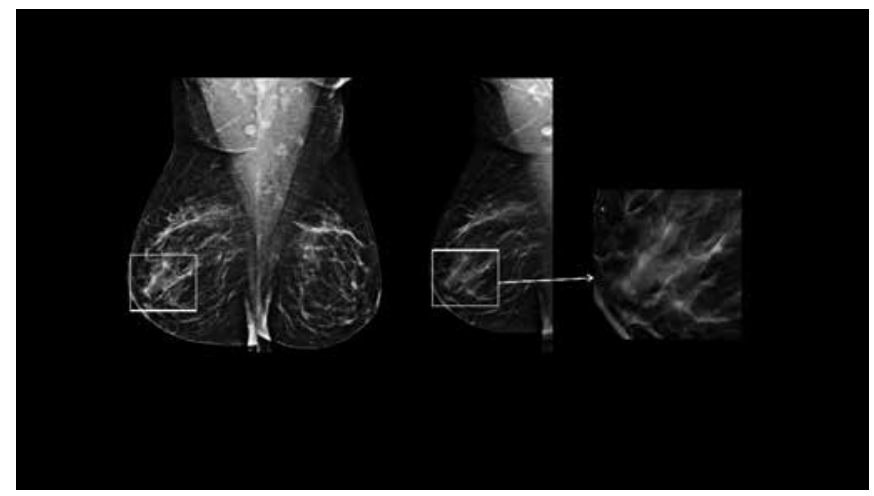

Fig. 11. Global asymmetry seen on RMLO view in upper outer quadrant of the right breast (red oval)

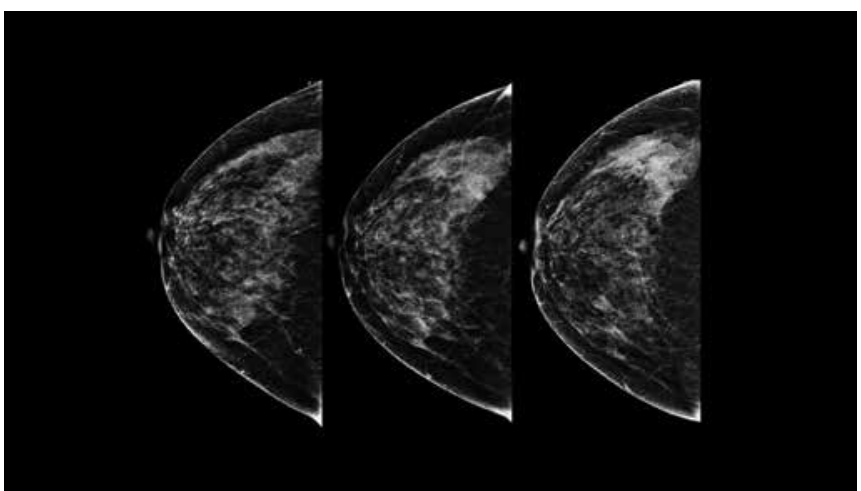

Fig. 13. RCC views show that in lateral quadrants (red oval) has developed over 2 years, larger and larger and more conspicuous asymmetry.

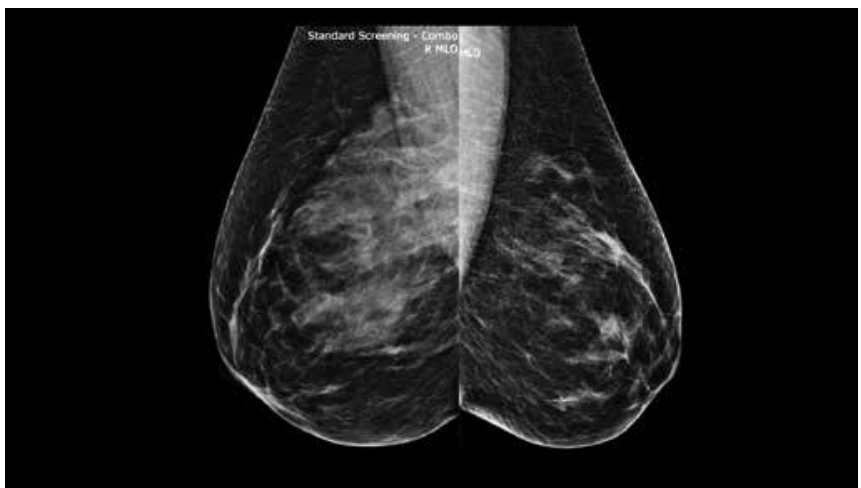

Fig. 8. RMLO FFDM, DBT and LMLO views - asymmetry is seen on the RMLO views (red square)

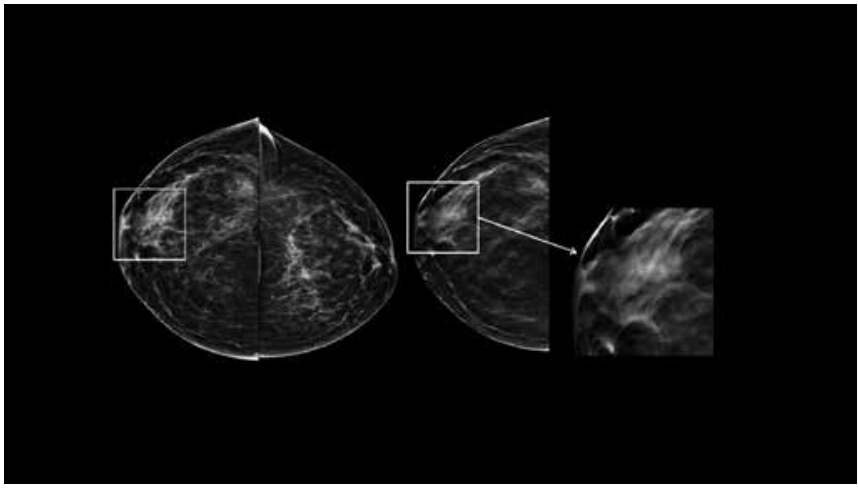

Fig. 10. RMLO FFDM, DBT and LMLO views - asymmetry is seen on the RMLO views (red square)

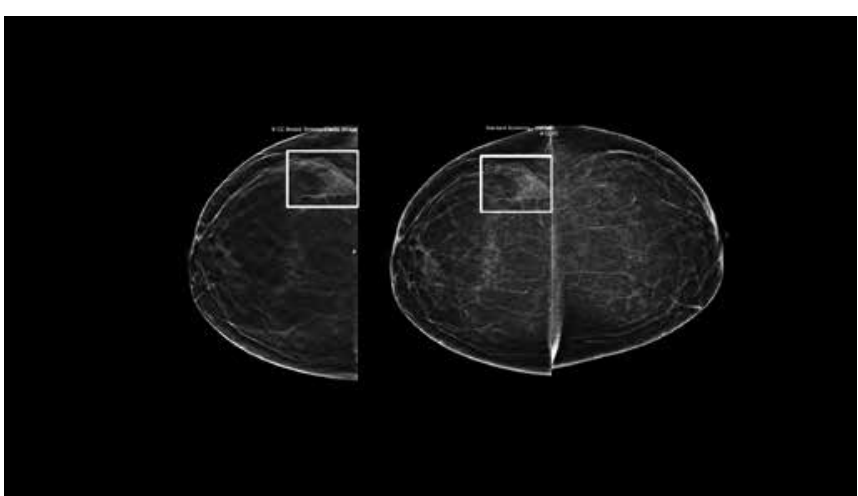

Fig. 12. Global asymmetry seen on RCC view in upper outer quadrant of the right breast (red oval)

as a normal variant $[18,20,25,26]$. In our study among 36 cases of global asymmetry no biopsies were performed and no breast cancers were detected during 24-28 months of follow-up after initial mammographic study.

Case Report №5. Global asymmetry seen on (Fig. 11) MLO and (Fig. 12) CC mammographic views in right upper outer quadrant of the right breast (red oval) represents a much greater volume of breast tissue relative to the corresponding region in the left breast with no associated mass, suspicious calcifications, or architectural distortion. Follow-up mammography in 12 and then 25 months from initial examination showed no interval change.

Developing asymmetry - is a focal asymmetry that is new, larger, or more conspicuous than on a previous ex- 
amination and occurs $0,11 \%-4,4 \%$ of all mammograms, predictive of cancer seen on $7,4 \%$ to $26,7 \%[15,18,19,20]$. This type of asymmetries can have benign (cyst, fibrocystic change, pseudoangiomatous stromal hyperplasia, scar, focal infections, weight loss or gain, trauma, fat necrosis, and hormone replacement therapy) and malignant causes invasive ductal and lobular cancers, invasive mucinous carcinoma and DCIS [19]. In our study we had 2 developing asymmetries, in both cases we performed CNB and revealed in first case DCIS and in second case radial scar associated with papilloma, typical ductal hyperplasia and sclerosing adenosis. Also case of DCIS associated with developing asymmetry was classified as false-negative due to diagnostic character of mammography.

Case Report №6. Developing asymmetry representing malignancy. (Fig. 13) RCC mammographic views show that in lateral quadrants (red oval) has developed over 2 years, larger and more conspicuous asymmetry than on previous examinations, now called a developing asymmetry. It was found to be ductal cancer in situ.

\section{CONCLUSIONS}

The results of our study suggest that DBT can better differentiate benign breast asymmetries, which are due to summation artifact, from breast cancers which may appear similar to normal fibroglandular tissue at FFDM. Depending on asymmetric finding, defined by BI-RADS atlas, we can presuppose the likelihood of cancer after mammographic examination.

In this study, developing asymmetry showed the highest rate of BC involvement (1/2), on the second place was focal $(6 / 82)$ and on the third place was one-view asymmetry $(1 / 81)$, among global asymmetries there were no breast cancers detected during two years of follow-up. Using of DBT in assessment of breast asymmetries can improve sensitivity and specificity in breast cancer detection and reduce number of unnecessary biopsies and short-interval follow-up examinations.

\section{REFERENCES}

1. Fedorenko Z.P., Michailovich Y.Y., Goulak L.O. et al. Cancer in Ukraine 2018 to 2019, incidence, mortality, activities of oncological service. In: Kyiv: National Cancer Institute of Ukraine. 2020: 42-43.

2. Broeders M., Moss S., Nyström L. et al. The impact of mammographic screening on breast cancer mortality in Europe: a review of observational studies.J Med Screen. 2012;19(1):14-25. doi:10.1258/jms.2012.012078.

3. Weedon-Fekjær H., Romundstad P.R., Vatten L.J. Modern mammography screening and breast cancer mortality: population study. BMJ. 2014; 348:3701.

4. Nyström L., Andersson I., Bjurstam N. et al. Long-term effects of mammography screening: updated overview of the Swedish randomised trials. The Lancet. 2002. doi.org/10.1016/50140-6736(02)08020-0.

5. Carney P.A., Miglioretti D.L., Yankaskas B.C. et al. Individual and combined effects of age, breast density, and hormone replacement therapy use on the accuracy of screening mammography. Ann Intern Med. 2003; 138:168-175. doi:10.7326/0003-4819-138-3200302040-00008.
6. Kolb T.M., Lichy J., Newhouse J.H. Comparison of the performance of screening mammography, physical examination, and breast US and evaluation of factors that influence them: an analysis of 27,825 patient evaluations. Radiology.2002;225:165-175. doi:10.1148/radiol.2251011667.

7. Kerlikowske K., Hubbard R.A., Miglioretti D.L. et al. Comparative effectiveness of digital versus film-screen mammography in community practice in the United States: a cohort study. Ann Intern Med. 2011;155(8):493-502.

8. Durand M.A., Haas B.M., Yao X. et al. Early clinical experience with digital breast tomosynthesis for screening mammography. Radiology. 2015; 274:85-92. doi: 10.1148/radiol.14131319.

9. Lourenco A.P., Barry-Brooks M., Baird G.L. et al. Changes in recall type and patient treatment following implementation of screening digital breast tomosynthesis. Radiology. 2015; 274:337-342. doi: 10.1148/ radiol.14140317.

10. Rose S.L., Tidwell A.L., Bujnoch L.J. et al. Implementation of breast tomosynthesis in a routine screening practice: an observational study. AJR Am J Roentgenol. 2013; 200:1401-1408. doi: 10.2214/AJR.12.9672.

11. Babkina T.M., Dykan I.M., Gurando A.V. et al. Detection of breast cancer presenting as a mass in women with dense breasts - digital breast tomosynthesis versus full-field digital mammography. Exp Oncol. 2020;42(3):215-219. doi:10.32471/exp-oncology.2312-8852.vol-42no-3.14898.

12. Dykan I.M., Bozhok Ye.M., Gurando A.V. et al. Cyfrovyi tomosyntez v diagnostytsi zahvoryuvan grudnyh zaloz; rozkish chy neobhidnist?(Analytychnyi oglyad literature) [Digital tomosynthesis in the diagnosis of breast diseases: luxury or necessity? (Analytical review of the literature)].Woman's Health.2017; 8(124):108-115. (in Ukrainian).

13. Samardar P., de Paredes E.S., Grimes M.M. et al. Focal Asymmetric Densities Seen at Mammography: US and Pathologic Correlation. 2002. doi.org/10.1148/radiographics.22.1. g02ja2219.

14. Peart J., Thomson G., Wood S. Developing asymmetry in a screening mammogram: A cautionary tale of a missed cancer. J Med Imaging Radiat Oncol. 2018;62(1):77-80. doi: 10.1111/1754-9485.12677.

15. Leung J.W., Sickles E.A. Developing asymmetry identified on mammography: correlation with imaging outcome and pathologic findings. AJR. 2007; 188: 667-75.

16. Hoff S.R., Abrahamsen A.L., Samset J.H. et al. Breast cancer: missed interval and screening-detected cancer at full-field digital mammography and screen-film mammography- results from a retrospective review. [Erratum appears in Radiology. $2013 \mathrm{Jan}$; 266(1):367]. Radiology 2012; 264:378-86.

17. Ciatto S., Houssami N., Ambrogetti D. et al. Minority report - false negative breast assessment in women recalled for suspicious screening mammography: imaging and pathological features, and associated delay in diagnosis. Breast Cancer Res Treat. 2007; 105:37-43.

18. Sickles E.A., D'Orsi C.J., Bassett L.W. et al. ACR BI-RADS mammography. In: ACR BI-RADS atlas Breast Imaging Reporting and Data System 2013. 5th ed. Reston, Va: American College of Radiology. 2013;1-175.

19. Chesebro A.L., Winkler N.S., Birdwell R.L. et al. Developing Asymmetries at Mammography: A Multimodality Approach to Assessment and Management. RadioGraphics.2010; 36:2. doi.org/10.1148/ rg.2016150123.

20. Venkatesan A., Chu P., Kerlikowske K. et al. Positive predictive value of specific mammographic findings according to reader and patient variables. Radiology. 2009;250(3):648-657. doi:10.1148/ radiol.2503080541. 
21. Dykan I.M., Bozhok Ye.M., Gurando A.V. Pershyi dosvid vykorystannia 3D mamohrafii v Ukraini. [The first experience of using 3D mammography in Ukraine]. Luchevaja diagnostika. Luchevaja Terapija. 2018; 2:40-8. (in Ukrainian).

22. Bozhok Ye.M., Gurando A.V. Imitatsiya raku lokalnoyu asymetriyeyu grudnoi zalozy. Klinichny vypadok. [Imitation of cancer by local asymmetry of the breast. Clinical case.]. Luchevaja diagnostika. Luchevaja Terapija 2017; 2:68-73. (in Ukrainian).

23. Sickles E.A. Findings at mammographic screening on only one standard projection: outcomes analysis. Radiology. 1998;208(2):471-475.

24. Sickles E.A. The spectrum of breast asymmetries: imaging features, work-up, management. Radiol Clin North Am. 2007;45(5):765-771.

25. Kopans D.B., Swann C.A., White G. et al. Asymmetric breast tissue. Radiology. 1989;171(3):639-643.

26. Youk J.H., Kim E.K., Ko K.H. et al. Asymmetric Mammographic Findings Based on the Fourth Edition of BI-RADS: Types, Evaluation, and Management. RadioGraphics. 2009; 29(1). doi.org/10.1148/rg. e33.

\section{ORCID and contributionship:}

Andrii V. Gurando: 0000-0002-2708-3040 B,D

Tetiana M. Babkina: 0000-0001-5046-870X ${ }^{A}$

Iryna M. Dykan: 0000-0001-8776-3278 ${ }^{E}$

Tetiana M. Kozarenko: 0000-0002-0838-9773 ${ }^{E}$

Viacheslav R. Gurando: 0000-0001-6303-3799 ${ }^{F}$

Vadim V. Telniy: 0000-0001-9860-9663 ${ }^{\mathrm{C}}$

\section{Conflict of interest:}

The Authors declare no conflict of interest.

\section{CORRESPONDING AUTHOR Andrii V. Gurando}

SI "Institute of Nuclear medicine and diagnostic Radiology of National Academy of Medical Science of Ukraine" 32 Platona Maiborody St., 04050 Kyiv, Ukraine, tel:+38(096) 273-92-73

e-mail: avgour@gmail.com

Received: 03.11 .2020

Accepted: 02.03.2021

A - Work concept and design, B - Data collection and analysis, C - Responsibility for statistical analysis, $\mathbf{D}$-Writing the article, $\mathbf{E}$-Critical review, $\mathbf{F}$ - Final approval of the article 